(Aus der Königl. Frauenklinik zu Dresden.)

\title{
Die vaginale Anwendung der Braun'schen Blase in der Geburtshülfe.
}

Von

Dr. Voigt, Assistenzarzt.

Wer den Braun'schen Kolpeurynter häufiger in der Geburtshülfe verwendet, wird die Erfahrung gemacht haben, dass derselbe bei vaginaler Anwendung meistens seinen Zweek in jeder Weise erfüllt, ab und zu aber versagt. Auch bei uns wurde dieses auffällige Imstichlassen zuweilen, jedoch mit zunehmender Erfahrung immer seltener beobachtet. Schon diese Thatsache weist darauf hin, dass ein Ausbleiben der Wirkung nicht dem Instrumente und der Methode als solchen zur Last fällt. Trotzdem wird die Braunsche Blase von Einigen überhaupt verworfen und an ihre Stelle der unelastische Ballon von Champetier empfohlen; von Anderen wird sie bei nur vaginaler Anwendung als in ihrer Wirkung durchaus unsicher bezeichnet und nur intrauterin eingelegt. Andere Veröffentlichungen beschäftigen sich nur mit der Verwendung and Brauchbarkeit des Braun'schen Kolpeurynters für besondere Fälle, z. B. bei Placenta praevia, bei Querlage, u. s. w. Die ausführlichste Arbeit über ihre Verwendung ist wohl die unter dem Titel: Enges Becken und Kolpeuryse erschienene von Rosenthal (Dieses Archiv, Bd. 45), in welcher der Verfasser auf Grund der bis zum Jahre 1895 in der Dresdener Königl. Franenklinik mit Kolpeuryse behandelten Fälle die Braun'sche Blase auch für die Praxis empfiehlt.

Auf Grund der guten Erfolge, die ich auf dem Gebärsaale mit der vaginalen Anwendung des Braun'schen Kolpeurynters erzielte, 
und auf Veranlassung meines hochverehrten Chefs, des Herrn Geheimrath Professor Dr. Leopold, unternahm ich eine Durchsicht aller der Fälle, die seit dem 1. 1. 1895 mit "Kolpeuryse" behandelt worden sind. An der Hand der 510 hier in Frage kommenden Fälle, die sich auf 12445 Geburten im Verlauf von 6 Jahren vertheilen, soll versucht werden, darzulegen, ob die Arbeit von Rosenthal einer Ergänzung bedarf, und welcher Art dieselbe sein müsste. Ferner soll gezeigt werden, dass zur erfolgreichen vaginalen Verwendung der Braun'schen Blase eine genaue Indicationsstellung, sowie die Erfüllung bestimmter Vorbedingungen nöthig ist. Es soll schliesslich im zweiten Theile der Einfluss der Kolpeuryse auf den Wochenbettsverlauf, sowie auf den Schutz des kindlichen Lebens unter der Geburt besprochen werden.

Die Vorbereitungen zum Einlegen der Braun'schen Blase sind ausführlich in der Rosenthal'schen Arbeit angegeben. Sie sollen deshalb hier nur kurz besprochen werden. Die Reinigung des K. geschieht genau wie die Desinfection der Hände, also durch Bürsten mit heissem Wasser und Seife, sowie darauf mit Sublimatlösung. Nach Entleerung der Harnblase und des Mastdarms wird die Scheide mit einer Sublimatlösung 1:4000 ausgespült und der durch etwas steriles Oel leicht gleitend gemachte Kolpeurynter unter Leitung des Zeigefingers in das hintere Scheidengewölbe eingeführt und dort mit demselben gehalten, während eine zweite Person den Ballon mittelst einer Stempelspritze mit sterilem Wasser oder, besonders in der Poliklinik, mit einer schwachen Lösung eines Desinficiens anfüllt. Es ist von Wichtigkeit, darauf zu achten, dass der Kolpeurynther "ganz prall" aufgespritzt ist, was sich leicht mit dem einführenden Finger feststellen lässt. Um je nach der Weite der Scheide dieselbe mit dem straff gefüllten Ballon ausfüllen zu können, sind bei uns 3 Grössen im Gebrauch, zu $300-400$; zu $450-550$; und zu $650-750 \mathrm{ccm}$. Zu empfehlen sind solche Kolpeurynter, die - je nach der Grösse von 1,5-2,5 mm Wandstärke - bei richtigem Füllungsgrade ihre Form auf Druck kaum verändern. Doch thut man gut, sich vor dem Einlegen noch einmal dadurch von der Dichtigkeit des Ballons zu überzeugen, dass man ihn bis zu der gewünschten Spannung anfüllt. Ist der Kolpeurynter in der Scheide soweit aufgespritzit, dass der Finger ihn eben eindrücken kann, so klemmt oder bindet man den Schlauch zu, um das Wiederausfliessen des eingespritzten Quantums zu verhindern. Der Kolpeurynter füllt dann, falls er in 
der Grösse richtig gewählt ist, die Scheide voll aus, ruht mit seinem unteren Ende auf dem Beckenboden und übt mit seiner oberen Wölbung einen Druck auf den Muttermund aus, wodurch das Scheidengewölbe entfaltet wird. Sollte der Kolpeurynter zu gross gewäblt worden sein, so lege man lieber an seinẹ Stelle eine kleinere Nummer ein; durch Ablassen eines Theiles der Flüssigkeit den zu starken Druck verringern zu wollen, bedeutet zugleich ein vollständiges Nutzlosmachen der Kolpeuryse, „denn nur als wenig nachgiebige, die Scheide voll ausfüllende Kugel erfüllt die Braun'sche Blase ihren Zweck!"

Die Beschwerden der Gebärenden sind, falls der Kolpeurynter die richtige Grösse hat, verhältnissmässig gering. Sie bestehen in einem Druck nach unten, ähnlich wie bei Stuhldrang; doch gewöhnen sich die Frauen bald daran. Ist eine Kreissende sehr empfindlich, so wirken schon $0,01 \mathrm{~g}$ Morph. subcutan beruhigend, ohne die Wehenthätigkeit ungünstig zu beeinflussen. Ist jedoch eine Kreissende sehr ungeberdig, oder der Introitus aus irgend einem Grunde sehr weit, so wird ein willkürliches oder unwillkürliches Auspressen des Kolpeurynters am besten dadurch verhütet, dass man die Kniee der Frau mit einem Tuche zusammenbindet. Auf diese Weise gelingt es meist, den Kolpeurynter selbst bei sehr kräftigen Wehen viele Stunden lang in der Scheide zu halten. Wie lange der Kolpeurynter in der Scheide liegen bleiben muss, bis man wieder zu untersuchen hat, hängt von der Art des Falles ab; im Allgemeinen kann man, wie bereits Rosenthal angiebt, früher als 3 Stunden nach Einlegen des Ballons keine wesentliche Aenderung des Befundes erwarten. Am meisten empfiehlt es sich, nach 6-8 Stunden die erste innere Untersuchung vorzunehmen. In dieser Zeit muss die Kolpeuryse schon gewirkt haben - wenn der Fall dafür geeignet ist -, andererseits kann sie bei besonders kräftiger Wirkung den weiteren Verlauf der Geburt noch nicht ungünstig beeinflusst haben. Zum Zweck der Untersuchung entfernt man die Braun'sche Blase nach Ablassen der Flïssigkeit und legt sie in eine Schüssel mit Sublimatlösung; ergiebt die Untersuchung, dass der Kolpeurynter noch nicht genügend gewirkt hat, so ist derselbe zum nochmaligen Einlegen ohne Weiteres fertig. Man thut gut, den Ballon erst zu entfernen, wenn alles zur Untersuchung bereit ist, um nicht während der Vorbereitung von Ereignissen überrascht zu werden, die ein sofortiges Eingreifen erfordern, wie z. B. Vorfallen der Nabelschnur. Sind die Wehen sehr kräftig; 
so Kann man neben dem in der Scheide liegenden Kolpeurynter untersuchen, nachdem man nur einen Theil seines Inhaltes abgelassen hat.

Die Wirkung des Kolpeurynters ist eine dreifache, wenn auch bald die eine, bald die andere mehr im Vordergrunde steht.

1. Erhaltung des Fruchtwassers, d.h. Schutz der Blase, oder bei gesprungener Blase, möglichste Einschränkung des Wasserabflusses.

2. Vorbereitung der Weichtheile, d. h. Eröffnung des Muttermundes, Auflockerung der Scheide und des Dammes.

3. Verstärkung der Wehen.

Zu dem letzten Punkte sei schon an dieser Stelle bemerkt, dass ein Erregen von Wehen nur dann möglich ist, wenn der Kolpenrynter sehr gefüllt wird und beträchtliche Zeit liegen bleibt (Ahlfeld). Da die Frau dabei anch in der Wehenpause ziemlich starken Schmerzen ausgesetzt ist, wird der Kolpeurynter bei uns zur Erregung von. Wehen nicht verwendet.

Die Dauer der Kolpeuryse ist naturgemäss in jedem Einzelfalle verschieden, doch thut man gut, die Braun'sche Blase nicht zu früh zu entfernen, da man sonst nicht selten ein Nachlassen der Wehen bemerkt. In vielen Fällen ist das Ausstossen des Kolpeurynters ein Zeichen dafür, dass er seinen Zweck erfüllt hat. Man findet dann bei der inneren Untersuchung, dass der Muttermund vollständig eröffnet ist und der Kopf im Becken steht. Es empfiehlt sich deshalb, den Kolpeurynter so lange in der Scheide liegen zu lassen, bis derselbe ausgestossen wird.

Von verschiedenen Seiten wird dem Kolpeurynter der Vorwurf gemacht, dass er den Kopf hindern soll, in und durch das Becken zu treten; dies ist jedoch weder theoretisch noch praktisch verständlich. Wenn auch obne Weiteres zuzugeben ist, dass er den Kopf aus dem Becken hebt, so karı man durch Hochbinden des Leibes und event. Fixiren des Kopfes über dem Beckeneingang zwischen zwei zusammengelegten Handtüchern ein Abweichen nach der Seite leicht verhüten. Ebensowenig als das Eintreten in das Becken wird eine günstige Einstellung des Kopfes durch den Kolpcurynter verhindert. Der Kopf drückt den Ballon auf den Beckenboden, erweitert dadurch die Vulva und dehnt den Damm. Während dieser Vorbereitung der Weichtheile steht der vorangehende Theil noch ziemlich hoch, presst dann aber den Kolpeurynter heraus, um der Regel nach fest in den Beckeneingang und meist sehr schnell tiefer zu treten. 
Bei engem Becken.

Bei der Betrachtung der Indicationen zur Anwendung des Kolpeurynters mögen die Fälle von engen Becken voranstehen, zumal sie ja auch der Zahl nach - 390 Fälle - bei weitem die erste Stelle einnehmen. Als enge Becken sind diejenigen gerechnet, welche eine C. d. von 10,5 abwärts haben; auf die C. v. ist deshalb nicht näher eingegangen, weil dieselbe nur schätzungsweise, je nach dem Stand des Promontoriums u. s. w. bestimmt werden kann. Gerade bei engen Becken kommt es erfahrungsgemäss besonders darauf an, die Blase so lange, wie möglich zu erhalten, damit das Kind bei dem ohnehin schweren Verlauf der Geburt durch vorzeitigen Verlust des Fruchtwassers nicht noch mehr gefährdet werde. Es wurde deshalb der Kolpeurynter in ausgedehntem Maasse bei den Fällen von E. B. zum Schutze der Fruchtblase verwendet. Bei bereits gesprungener Blase ist es aus demselben Grunde stets von Wichtigkeit, möglichst viel Fruchtwasser noch zu erhalten. Dies besorgt die Braun'sche Blase, indem sie die Scheide ausfüllt und ein weiteres Abfliessen desselben verhindert. Ausserdem übernimmt sie an Stelle der gesprungenen Fruchtblase deren Function. Da bei beweglich stehendem Kopfe und E. B. das Vorfallen der Nabelschnur oder kleiner Theile nicht eben selten ist, bringt das Einlegen des Kolpeurynters weiterhin den Vortheil mit sich, dass es eine solche Gefahr für das Kind sowohl bei noch erhaltener, wie bereits gesprungener Blase verhütet. Indem dio Braun'sche Blase einerseits einen vorzeitigen Blasensprung verhütet, hilft sie indirekt zur Vorbereitung des Muttermundes, andererseits wirkt sie - auch bei gesprungener Blase - durch Verstärkung der Wehen in gleichem Sinne. - Man wird deshalb bei E. B. den Kolpeurynter überall anwenden, wenn eine der oben beschriebenen Wirkungen, oder, wie es die Regel ist, alle zusammen erwünscht sind. Das wäre also bei Erstgebärenden mit hoch und beweglich stehendem Kopf sowohl bei erhaltener, wie bei bereits gesprungener Blase. Sind bei Mehrgebärenden nach dem Verlauf der früheren Geburten wieder schwere Entbiodungen zu erwarten, so empfiehlt es sich, den Kolpeurynter dann einzulegen, wenn der Kopf bei stehender oder bereits gesprungener Blase noch beweglich ist. Doch kann man sich auch in den Fällen, wo der Kopf fest im Beckeneingang oder demselben aufgepresst steht, selbst bei noch erhaltener Blase, mit Vortheil des Kolpeurynters bedienen, da auch bei dem weiteren Durchtreten 
des Kopfes durch das E. B. das kindliche Leben noch stark gefährdet ist. Auf diese. Weise kann die volle Wehenkraft auf die Configuration des Kopfes verwendet werden, während der Kolpeurynter schon rein mechanisch demselben den Weg durch die Weichtheile vorbereitet. Aus dem gleichen Grunde empfiehlt es sich, in Fällen von Kolpeuryse bei beweglich stehendem Kopf, die Braun'sche Blase so lange in der Scheide liegen zu lassen, bis der tieferrückende Kopf oder die sich vorwölbende Fruchtblase dieselbe herausdrängen oder bei vollständigem Muttermunde eine weitere Wirkung derselben nicht mehr zu erwarten oder erwünscht ist. Es wird dann in dem einen Fall besonders der Schutz bezw. Ersatz der Blase, in dem andern mehr die Verstärkung der Wehen, in einem dritten besonders die Vorbereitung der Weichtheile erwartet, immer abor sind die andern Wirkungen solche, welche die Geburt fördern.

Auf diese Weise wurde in 239 Fällen bei engem Becken eine Spontangeburt erzielt. Von diesen Frauen waren 133 Erstgebärende, von denen 83 eine C. d. von 10,5-9,6 hatten, 50 dagegen eine solche von 9,5 abwärts. Beim Einlegen des Kolpeurynters war bei ersteren in 50 Fällen die Blase noch erhalten, bei 33 dagegen schon vorher gesprungen. In der zweiten Gruppe war die Blase bei 36 Frauen noch erhalten, während sie bei 14 bereits fehlte. Die Zahl der Mehrgebärenden mit engem Becken, die nach Kolpeuryse spontan niederkamen, beträgt 106. Bei 73 von ihnen betrug die C. d. 10,5-9,6, bei den übrigen 33 weniger. Der Unterschied der. Kolpeurysen bei erhaltener oder bei gesprungener Blase ist hier gering; es stellt sich für die erste Gruppe das Verhältniss auf $42: 31$, für die zweite auf $17: 16$. Die Beobachtung, dass bei Mehrgebärenden überhaupt, besonders aber bei den Becken mit einer C. d. von 9,5 abwärts weniger Spontangeburten erzielt wurden, ist für die Leitung der Geburt bei engem Becken von Bedeutung. Während bei Mehrgebärenden die Enge des Beckens nicht selten eine Indication zum Eingreifen bildet, so wird wegen des engen Beckens allein — von den Fällen mit hochgradigster Verengerung abgesehen - bei Erstgebärenden nicht zu einer Operation geschritten.

Bei der Betrachtung der Operationen, welche bei Gebärenden mit engem Becken ausgeführt wurden, muss streng unterschieden werden zwischen den durch das enge Becken bedingten und solchen, für welche andere Verhältnisse die Indication bildeten. 
Wendungen aus anderen Gründen, als wegen Nichteintretens des Kopfes sind im Folgenden der Vollständigkeit halber zwar mit angeführt, bei der Zusammenstellung der Operationen wegen engen Beckens aber unberücksichtigt geblieben.

Nach Zahl und Art zeigen die Operationen bei Erst- und Mehrgebärenden ganz erhebliche Unterschiede. So ist die Wendung wegen Nichteintreten des Kopfes eine Operation, die, zu rechter Zeit vorgenommen, bei Mehrgebärenden oft ein sonst verlorenes kindliches Leben zu retten ermöglicht. Bei Erstgebärenden ist diese Operation fast aussichtslos; sie wurde deshalb nur $6 \mathrm{mal}$ versucht, und zwar $2 \mathrm{mal}$ bei einer C. d. von $10,5-9,6$, je 1 mal bei noch erhaltener und bei gesprungener Blase and 4 mal bei einer C. d. unter 9,6. Die Resultate waren, wie weiter unten ausgeführt werden wird, wenig erfreulich. Da eine Querlage bei Erstgebärenden am Ende der Schwangerschaft überhaupt sehr selten ist, wurde auf Grund der Querlage bei Erstgebärənden mit engem Becken nur zweimal die innere Wendung vorgenommen, einmal bei einer C. d. von 10,5-9,6 und einmal unter 9,6. In beiden Fällen war es durch die Kolpeuryse gelungen, die Blase bis zu Beginn der Operation zu erhalten.

Falls der kindliche Kopf das Becken nicht passiren kann, aber dringende Indication zu baldiger Beendigung der Geburt vorliegt, kommen nur die Anlegung der Zange oder die Perforation in Frage. Für beide Operationen schaffit der Kolpeurynter die günstigsten Vorbedingungen. Die Vorbereitung der Weichtheile und die Eröffnung des Muttermundes ist bei rechtzeitigem Einlegen der Braun'schen Blase zu der Zeit, da die Operation angezeigt ist, in den allermeisten Fällen erreicht und der Kopf ist fest in den Beckeneingang getreten. Es wird also die ohnehin nur selten nöthig werdende Anwendung der Zange bei oberhalb der Beckenmitte stehendem Kopfe wesentlich erleichtert und dadureh etwas aussichtsvoller gemacht. Meistens stand zu der Zeit des Eingriffes der Kopf aber wesentlich tiefer im Becken, so dass man zu jener Operation bei Erstgebärenden nur 5 mal seine Zuflucht zu nehmen brauchte. Hierbei hatte in 4 Fällen das Becken eine C. d. von 10,5-9,6, einmal eine noch geringere. Der Kolpeurynter war bei 4 Frauen bei noch nicht gesprungener Blase, einmal erst nach dem Blasensprung eingelegt worden. Die Anwendung der Zange bei im Beckeneingang stehendem Kopfe, um theils im Interesse des Kindes, theils zur Schonung der Mutter wegen der drohenden In- 
fection die Geburt zu beendigen, war in 10 Fällen angezeigt, und zwar nach Kolpeuryse bei noch erhaltener Blase nur $3 \mathrm{mal}$; die C. d. betrug hier stets weniger als 9,6! Bei den übrigen Fällen war die Blase bereits vorzeitig gesprnngen; 6 mal betrug die C. $d$. $10,5-9,6,1 \mathrm{mal}$ weniger.

Im Vergleich zu den Zangenoperationen. sind die Perforationen allerdings zahlreicher, wenn auch ihr Verhältniss zu der Zahl und Art der engen Becken mit Recht als ein sehr günstiges bezeichnet werden muss. Ein wesentlicher Unterschied in der Hänfigkeit bei den Geburten Erst- oder Mehrgebärender ist bei dieser Operation nichit zu erkennen. Der Kolpeurynter schafft auch für diesen Eingriff durch Erweitern von Muttermund und Scheide, sowie durch die Verstärkung der Wehen, die den Kopf wenigstens mit einem kleinen Segment in den Beckeneingang pressen, günstige Vorbedingungen. So wird die schwierige und für die Mutter nicht ungefährliche Perforation bei engem Muttermund und beweglichem Kopf fast stets vermieden. Von den 30 Frauen, bei denen die Perforation angezeigt war, betrug die C. d. 8 mal $10,5-9,6$, bei den anderen noch weniger. In 17 Fällen war die Blase beim. Einlegen des Kolpeurynters noch erhalten, 13 mal fehlte sie bereits.

Betrachtet man nun die Art und Zahl der Operationen bei Mehrgebärenden mit engem Becken und vergleicht sie mit dem Vorhergehenden, so ergiebt sich für die Perforation kein wesentlicher Unterschied; es soll deshalb diese Operation bei der Besprechung vorangestellt werden. Bei 22 Mehrgebärenden musste die Geburt durch Perforation beendigt werden, und zwar $10 \mathrm{mal}$ bei einer C. d. von 10,5-9,6 und 12 mal bei einer solchen von 9,5 abwärts. In 12 Fällen war die Blase beim Einlegen des Kolpeurynters noch erhalten gewesen, bei den übrigen 10 fehlte sie bereits. Auch hier stellt sich das Verhältniss ähnlich wie bei den Erstgebärenden. - Zangenoperationen waren bei Mehrgebärenden mit engem Becken nur $7 \mathrm{mal}$ angezeigt, und zwar stand nur $1 \mathrm{mal}$ bei einer C. d. von 10,5 der Kopf noch nicht in der Beckenmitte; in den übrigen 6 Fällen war er bereits tiefer getreten und es wurde je $3 \mathrm{mal}$ bei einer C. d. von 10,5-9,6 und unter 9,6 operirt.

Unter den durch das enge Becken bei Mehrgebärenden bedingten Operationen überwiegt die Wendung aus Kopflage sowohl absolut wie relativ alle anderen Eingriffe bei Mehr- wie bei Erstgebärendẹn in hohem Grade. Diese Thatsache wird durch die Leitung der Geburt zum Theil erklärt. Ist bei einer Mehrgebären- 
den mit beweglich stehendem Kopf nach dem Verlauf der früheren Geburten eine schwere Entbindung zu erwarten, so wird, wie oben erwähnt, der Kolpeurynter eingelegt, womöglich bevor die Blase springt. Ist dann bei vollständig eröffnetem Muttermund der Kopf nicht eingetreten, so wird die Blase gesprengt und abgewartet, ob derselbe dann eintritt. Ist dies nicht im Verlauf von 1-2 Stunden geschehen, so wird zur Wendung geschritten. Oder man benutzt die durch den Kolpeurynter geschaffenen günstigen Verhältnisse, vollständig eröffneten Muttermund und erhaltene Blase, um gleich die Wendung vorzunehmen. Unter den 58 Wendungen wegen Nichteintretens des Kopfes bei Mehrgebärenden gelang die Erhaltung der Blase bis zur vollständigen Eröffnung des Muitermundes in 54. Fällen, während 4 mal die Blase vorzeitig gesprungen war. Nach der Enge des Beckens vertheilen sich diese Wendungen derart, dass $22 \mathrm{mal}$ bei einer C. d. von $10,5-9,6$ und $36 \mathrm{mal}$ bei einer solchen von 9,6 und weniger operirt wurde.

Eine Querlage bildete bei Mehrgebärenden mit engem Becken in 11 Fällen die Anzeige zur Vornahme der Wendung. Hier wurde der Kolpeurynter wie bei einfachen Querlagen in der Absicht eingelegt, um für die Vornahme der Operation die günstigsten Vorbedingungen zu schaffen. So gelang es denn auch in allen 8 Fällen, wo die Blase noch unverletzt gefunden wurde, dieselbe bis zu Beginn der Operation zu erhalten. In 3 Fällen war die Blase bereits vorzeitig gesprungen, doch erreichte man auch hier durch die Kolpeuryse vollständige Eröffnung des Muttermundes und möglichste Erhaltung des Fruchtwassers. Bei diesen Wendungen hatten 4 Becken eine C. d. von 10,5-9,6 und 7 eine geringere.

Wenn es sich auch nicht mit Zahlen beweisen lässt, dass. einzelne Geburten bei engen Becken auch ohne die Anwendung. der Braun'schen Blase spontan verlaufen wären, so spricht doch. das Verhältniss der Spontangeburten zú der Zahl der operativen. Entbindungen bei engen Becken für die günstigen Wirkung des. Kolpeurynters.

Bei normalen Becken.

Tiefer Sitz der Placenta mit Blutung und Placenta praevia bildeten im Ganzen $14 \mathrm{mal}$ die Indication zum Einlegen des Kolpeurynters. Man beabsichtigte dabei erstens mit ihm bei noch nicht gesprengter Blase zu tamponiren, ferner aber auch die Wehen zu verstärken und die Weichtheile vorzubereiten. Auf 
Grund der in diesen Fällen gemachten Erfahrungen ist hervorzuheben, dass durch das Einlegen der Braun'schen Blase in die Scheide die Blutung nicht mit Sicherheit gestillt wird, sondern zur Tamponade sterile Gazestreifen oder Wattekugeln entschieden zuverlässiger sind. Immerhin gelang es in einigen Fällen auch so, die Blutung zum Stehen zu bringen oder doch in mässigen Grenzen zu halten. Während in einigen Fällen die Geburt dann spontan verlief, so wurde in anderen doch noch ein operatives Eingreifen nöthig. Aber sowohl für die combinirte, wie für die innere-Wendung, als für das Einlegen eines Metreurynters - wie es in den letzten Jahren geübt wurde — schaffte die vorhergegangene Kolpeuryse günstige Vorbedingungen.

Bei Eklampsie, die 8 mal mit engem Becken complicirt war, wurde der Kolpeurynter 19 mal vaginal angewendet, in der Absicht, durch Verstärkung der Wehen und Vorbereitung der Weichtheile die Geburt nach Möglichkeit zu beschleunigen. Ein Schützen der Blase, bezw. Erhalten des Fruchtwassers wurde in diesen Fällen weniger angestrebt, man hatte im Gegentheil bei einigen Frauen vorher schon die Blase gesprengt und so eine Beschleunigung der Geburt berbeizuführen versucht. Die Wehen nahmen denn auch nach dem Einlegen der Braun'schen Blase regelmässig an Stärke und Ausgiebigkeit zu, der Muttermund erweiterte sich in verhältnissmässig kurzer Zeit und es konnten so ohne andere Eingriffe noch einige Spontangeburten erzielt werden. Gestattete die Gefahr für das Leben der Mutter kein längeres Zuwarten, so musste operativ eingegriffen werden. Dass es in diesen Fällen nicht gelang, durch die Kolpeuryse die Operationen zu vermeiden, kann dem Instrument gewiss nicht zum Vorwurf gemacht werden. Es hat in diesen Fällen für dieselben wenigstens möglichst günstige Vorbedingungen gesehaffen. In 4 Fällen von schwerer Nephritis mit allen. Folgeerscheinungen wurde auf Grund derselben Indicationen, wie bei Eklampsie, der Kolpeurynter zur Beschleunigung der Geburt eingelegt. In 2 Fällen wurde so ohne Schädigung der Mutter eine Spontangeburt erzielt, in den beiden anderen nahmen die bedrohlichen Erscheinungen derart zu, dass zu möglichst schneller Eröfinung des Muttermundes die Braun'sche Blase intra-uterin eingelegt und mit dauerndem Zug versehen werden musste, sobald sich der Muttermund auf 2-3 Markstückgrösse erweitert hatte.

Zum Schutz der Blase und zur Vorbereitung der Weichtheile, 
weniger zur Verstärkung der Wehen wurde der Kolpeurynter eingelegt in 6 Fällen von Gesichtslage, die 5 mal mit engem Becken complicirt waren und 8 mal wegen Stirnlage bei engem Becken. Wie aus der ungünstigen Einstellung des Kopfes erklärlich, sind hier Operationen verhältnissmässig häufiger nöthig geworden. Bei den Gesichtslagen wurden 5 Spontangeburten erzielt, einmal - es handelte sich um eine ungünstige Einstellung mit dem Kinn nach hinten - wurde die Rettung des Kindes durch Wendung und Extraction versucht, doch gelang es nicht, den nachfolgenden Kopf. unzerkleinert durch das Becken zu leiten. Bei den 8 Fällen von Stirnlage erfolgte nach der Kolpeuryse die Geburt 4 mal spontan.

Der Verlauf eines dieser Fälle zeigt zugleich, dass der Kolpeurynter eine günstige Einstellung des Kopfes nicht hindert:

27jähr. Ilpa, Becken: 24, 26, 34, 191/2, 10 (8). 1. Abort, 2. Perf.Port. verstrichen, Mm. dreimarkstückgross, Blase fehlt, Stirilage; Kolpeurynter $550 \mathrm{ccm}$ eingelegt, wird nach $4 \frac{1}{2} 2$ Stunden ausgepresst, $1 / 2$. Stunde später Spontangeburt in Hinterhauptslage. Kind $52 \mathrm{~cm}$, $3680 \mathrm{~g}$.

Einmal musste das absterbende und einmal das todte Kind zur Schonung der Mutter perforirt werden, zweimal gelang es, das Kind durch Wendung und Extraktion zu entwickeln.

Dreimal wurde bei normalem Becken der Kolpeurynter eingelegt, um für die innere Wendung aus Querlage möglichst günstige Vorbedingungen zu schaffen, nachdem die äussere Wendung wiederholt vergeblich versucht worden war. Es gelang in allen Fällen, die Blase bis zur vollständigen Eröffnung des Muttermundes zu erbalten.

Besonders in die Augen fallend war die Wirkung des Kolpeurynters bei alten Erstgebärenden (12 Fälle), bei denen er besonders in der Absicht eingelegt wurde, um die engen und wenig nachgiebigen Weichtheile aufzulockern und für den Durchtritt des Kopfes vorzubereiten. Es gelang denn auch in allen Fällen auf diese Wcise trotz der Grösse der Kinder, bedeutendere Zerreissungen zu verhüten. Nicht so wirksam zeigte sich, wie schon Rosenthal beobachtete; die Braun'sche Blase bei der vaginalen Anwendung zur Dehnung von Narben am Muttermunde; in 3 Fällen musste sie deshalb intra-uterin eingelegt und mit elastischem Zug versehen zur Eröffnung des narbigen Muttermundes dienen. Zweimal genügte indessen der in die Scheide gelegte Ballon zur Auflockerung 
des Narbengewebes an der Portio. Zur Auflockerung und Dehnung von Kolporrhaphienarben erwies sich dagegen der Kolpeurynter in einem Falle als sehr geeignet und wäre für ähnliche Fälle zu empfehlen.

In mehreren Fällen war eine Beschleunigung der Geburt im Interesse des Kindes geboten, und es war wiederum der Kolpeurynter, der durch die Verstärkung der Wehen sowie die Vorbereitung der Weichtheile es in allen Fällen ermöglichte, das gefährdete kindliche Leben zu retten. Einmal war beim Blasensprung die. Nabelschnur vorgefallen, als der noch wenig geöffnete Muttermund eine Wendung und Extraction zur Rettung des Kindes noch nicht gestattete. Nach der Reposition der Nabelschnur wurde der Kolpeurynter eingelegt, die Wehen wurden kräftiger, der ins Beeken tretende Kopf drängte den Ballon heraus und die Entwicklung des gefährdeten Kindes mit dem Forceps gelang bei vollständig geöffnetem Muttermunde ohne Schwierigkeiten. Einmal wurde wegen starker Unregelmässigkeit der kindlichen Herztöne bei stehender Blase und noch nicht vollständig eröffnetem Muttermunde der Kolpeurynter eingelegt; die Herztöne erholten sich wieder und das Kind wurde, nachdem der Kolpeurynter ausgepresst worden war, lebensfrisch geboren. Es hatte in diesem Fall wohl eine Nabelschnurschlinge neben dem Kopf gelegen, wenn dieselbe auch nicht zu fühlen war. - - Sie war zurückgesunken, als der Ballon den Kopf in die Höhe drängte, und wurde so vor weiterem Druck bewahrt. Ein zweites Mal war eine in der. Blase liegende Nabelschnurschlinge deutlich gefühlt worden, es gelang aber nicht, dieselbe durch Lagerung zum Zurücksinken zu bringen; der Kolpeurynter bewirkte eine schnelle Erweiterung des Muttermundes und ermöglichte es, das Kind durch Wendung und Extraktion $\mathrm{zu}$ retten.

Wegen vorzeitigen Blasensprunges bei noch beweglich stehendem Kopf wurde bei 6 Frauen mit normalem Becken der: Kolpeurynter eingelegt, da die Wehenthätigkeit schlecht war und beständig Fruchtwasser absickerte und ausserdem noch die Gefahr des Vorfalls der Nabelschnur oder kleiner Theile bestand. Die Wirkung der Braun'schen Blase entsprach den bei engen Becken gemachten Erfahrungen und alle 6 Kinder wurden lebensfrisch und spontan geboren.

Neben diesen guten Erfolgen der Kolpeuryse, welche, abgesehen von den 3 Fällen von narbiger Stenose des Muttermundes, 
bisher stets den gehegten Erwartungen entsprach, muss es auffallen, dass auch in der Klinik eine allerdings geringe Zahl von Fällen beobachtet wurde, bei denen der gewünschte Erfolg überhaupt oder doch zunächst ausblieb. Da aber ein Vergleich dieser Fälle mit den vorhergehenden besonders gut erkennen lässt, dass die Kolpeuryse genau so wie jede andere geburtshülfliche Operation nur auf Grund einer richtig gestellten Indication vorgenommen werden sollte und dass zu ihrem Gelingen '- d. h. um die gewünschte Wirkung zu erzielen - bestimmte Vorbedingungen erfüllt sein müssen, mögen zunächst einige charakteristische Fälle in Kürze angeführt werden, aus denen sich die Regeln für die Anwendung der Braun'schen Blase ergeben.

1. 22jähr. Ipa. Becken $22,5,25,5,30,18$. Angeblich seit mehreren Stunden leise Wehen. Portio zapfenförmig, Mm. für die Fingerspitze zugängig. Kolpeurynter mit $400 \mathrm{ccm}$ eingelegt. Nachdem der Kolpeurynter im Ganzen 15 Stunden gelegen hatte, während welcher die Frau schmerzhafte Zusammenziehungen - jedoch keine Wehen - hatte, fand man die Portio etwas mehr aufgelockert und den Mm. für einen Finger durchgängig; der sonstige Befund war unverändert. Es wurde nun der Kolpeurynter nicht wieder eingelegt und die Zusammenziehungen hörten alsbald auf. Nach mehrstündiger Beobachtung wurde die Frau wegen Wehenlosigkeit vom Kreisssaale entlassen. 5 Tage später kam sie mit fest im Becken stehendem Kopf und Wehen wieder und gebar nach 6 Stunden spontan ein Kind von $54 \mathrm{~cm}$ und $4020 \mathrm{~g}$.

2. 21jähr. IIpa. Becken: 22,5, 24,5, 29,5, 17, 10 (8) mit schmerzhaften Zusammenziehungen; Portio wulstig, $\mathbf{M m}$. für die Finger gut durchgängig. Kolpeurynter eingelegt und mit $500 \mathrm{ccm}$ gefiullt. Der Kolpeurynther liegt im Ganzen 16 Stunden, ohne eine Aenderung. herbeizuführen und wird deshalb entfernt. 37 Stunden später beginnen leise Wehen, Portio wulstig, $\mathrm{Mm}$. zweimarkstückgross. Kolpeurynter eingelegt, $350 \mathrm{cem}$. Nach 6 Stunden Muttermund fünfmarkstückgross, Kolpeurynter wieder eingelegt, wird nach 4 Stunden ausgepresst, die Blase springt, der Kopf tritt fest in den Beckeneingang, ca. 1 Stunde später erfolgt die Spontangeburt eines Kindes von $49 \mathrm{~cm}$ und $2950 \mathrm{~g}$.

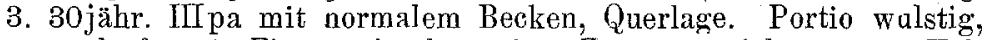
Muttermund für 1 Finger durchgängig, Zusammenziehungen. Kolpeurynter eingelegt mit $400 \mathrm{cem}$; nach 18 Stunden fortgelassen, da dauernd keine richtigen Wehen. 7 Stunden später treten erst Wehen auf, Portio wulstig, Muttermund zweimarkstückgross. Kolpeurynter eingelegt mit $450 \mathrm{ccm}$ gefüllt, wird nach $1 / 2$ Stunde ausgepresst, Blasensprung, Muttermund vollständig; nach $1 / 2$ Stunde Geburt, Kind $52 \mathrm{~cm}$, $3390 \mathrm{~g}$, in vollkommener Fusslage geboren.

In 30 Fällen wurde so der Kolpeurynter eingelegt, ohne dass die Geburt zunächst in Gang kam; es traten zwar wehenartige Schmerzen auf, aber keine richtigen Wehen und der Muttermund öfnete sich nicht. Die meist nach 6 Stunden vorgenommene innere 
Untersuchung ergab, abgesehen von einer Auflockerung der Vagina und Portio, keine wesentliche Aenderung des Befundes. Auch bei nochmaliger Kolpeuryse änderten sich die Verhältnisse nicht, so dass man von einer weiteren Verwendung der Braun'schen Blase zunächst absah. War eire baldige Beendigung der Geburt angezeigt, so wurde der Ballon intrauterin eingelegt und mit elastischem Zuge versehen; die'vorhergegangene Kolpeuryse erleichterte diesen Eingriff, da sie Portio und Muttermund aufgelockert und dehnbar gemacht hatte. In den anderen Fällen wartete man zunächst das Eintreten von richtigen Wehen ab und legte in dann noch geeignet erscheinenden Fällen von Neuem die Braun'sche Blase in die. Scheide und erzielte dann auch den gewünschten Erfolg.

Das Versagen der Braun'schen Blase in diesen Fällen zeigt, dass der auf Grund richtiger Indicationsstellung eingelegte Kolpeurynter seine Wirkung nicht entfalten kann, wenn nicht ganz bestimmte Torbedingungen erfüllt sind. Es bestätigt sich also zunächst die von Rosenthal gemachte Beobachtung, dass die Wehenthätigkeit wirklich begonnen haben muss, damit der Kolpeurynter das leisten kann, was man bei seiner Anwendung beabsichtigt und erwartet. Es darf ferner keine narbige Stenose des Muttermundes bestehen, die der Eröffnung desselben einen zu grossen Widerstand entgegensetzt. Ist der Muttermund nicht wenigstens markstückgross und die Portio bei Erstgebärenden noch nicht verstrichen, bei Mehrgebärenden wenigstens stark verkürzt, so wird der Kolpeurynter zunächst nur der Frau Beschwerden machen, ohne ihr im Geringsten zu nutzen; es empfiehlt sich deshalb, den Ballon erst dann einzulegen, wenn Portio und Muttermund erkennen lassen, dass eine regelrechte Wehenthätigkeit bereits vorhanden ist.

Zweimal erfüllte der Kolpeurynter seinen Zweck nicht ganz, obgleich er auf Grund richtiger Indication und mit Rücksicht auf die erfüllten Vorbedingungen eingelegt worden war, indem es nicht gelang, die Blase bis zur vollständigen Eröffnung des Muttermundes zu erhalten. In dem einen Falle ist aus dem Journal kein Grund für den Blasensprung, während der Kolpeurynter in der Scheide lag, zli ersehen, das andere Mal lag die Schuld wohl daran, dass der eingelegte Ballon für die Scheide zu klein gewählt worden war. In Bezug auf die Verstärkung der Wehen und die Vorbereitung der Weichtheile entsprach er den auf ihn ge- 
setzten Erwartungen. Im ersten Falle handelte es sich um eine Schädellage und es wurde trotz des vorzeitigen Blasensprunges noch eine Spontangeburt erzielt, da der Kopf gleich darauf fest in den Beckeneingang trat; im zweiten Falle erreichte man durch nochmaliges Einlegen des Kolpeurynters eine vollständige Eröffnung des Muttermundes und Erhaltung des Fruchtwassers, so dass die innere Wendung aus Querlage ohne Schwierigkeiten ausgeführt werden konnte.

Es ist also, wie sich aus dem Vorhergehenden ergiebt, die vaw ginale Anwendung der Braun'schen Blase durchweg von günstigem Einfluss auf den Verlauf der Geburt. Weiterbin ist nun von Interesse, zu sehen, ob im Verlauf des Wochenbetts eintretende Störungen mit ihrer Anwendung in Zusammenhang zu bringen sind.

Bei der Betrachtung, des Wochenbettsverlaufes müssen zunächst 5 Fälle ausgeschlossen werden, in denen die Frauen kurz nach der Entbindung starben. Bei drei Todesfällen an schwerer Eklampsie dürftewohl dem Kolpeurynter keineSchuld an dem unglücklichen Ausgange beizumessen sein. Ebensowenig bei dem Tod einer Frau mit schwerer Nephritis an atonischer Nachblutung. Als fünfter wäre einer der oben erwähnten Fälle von narbiger Stenose des Muttermundes zu nennen, wo die Kolpeuryse ohne Erfolg blieb. Es wurde hier, nachdem der Muttermund durch intra-uterine Ballonbehandlung mit elastischem Zug auf Dreimarkstückgrösse erweitert worden war, die combinirte Wendung gemacht, da die Frau möglichst schnell entbunden werden musste. Es kam trotz darauf folgender Exenteration und Perforation des nachfolgenden Kopfes eine Uterusruptur zu Stande, und trotz sofortiger Naht ging die Frau an acuter Anämie zu Grunde. Auch hier dürfte dem Kolpeurynter wohl keine Schuld beizumessen sein.

Von den übrig bleibenden 505 Wöchnerinnen machten 362 ein völlig ungestörtes Wochenbett durch. Als untere Grenze des Fiebers im Wochenbett wird für die Statistik $38,0^{\circ} \mathrm{C}$. angesehen, wenn auch Steigerungen von $37,6^{\circ} \mathrm{C}$. an, in der Achselhöhle gemessen, nicht mehr als normal betrachtet werden. Um möglichst zuverlässige Resultate zu erhalten, ist schon seit Jahren in der Dresdener kgl. Frauenklinik die Einrichtung getroffen, dass bereits $1 / 2$ Stunde vor der Visite die Thermometer gesteckt werden. Die Hebammenschülerinnen lesen dann die Temperatur ihrer Wöchnerinnen $a b$ und schreiben dieselbe auf die am Kopfende des Bettes 
befindliche Tafel. Das Thermometer bleibt jedoch noch in der Achselhöhle liegen und der Hülfsarzt, welcher die Vorvisite macht, controlirt bei jeder der ihm zugetheilten Wöchnerinnen die Temperatur; scheint ihm das Ergebniss nicht zutreffend, so lässt er die betreffende Wöchnerin noch einmal messen. Schliesslich werden etwaige. Unrichtigkeiten der Messung von dem Assistenten durch nochmaliges Einlegen des Thermometers richtig gestellt. So wird die grösste Sorgfalt auf das Beobachten der Temperaturen verwandt und eine Fiebersteigerung wohl kaum übersehen. Am 10. oder 11. Tage werden dann die Frauen, welche ein ungestörtes Wochenbett durchgemacht haben, entlassen; nur wenn ihr Krättezustand ein längereș Verweilen in der Anstalt wünschenswerth erseheinen lässt, wird ihre Entlassung, auch ohne dass sie gefiebert hätten, einige Tage hinausgeschoben.

Bei der Betrachtung der mit Fieber verlaufenden Puerperien sind zunächst 12 Fälle auszuschalten, in denen die Temperatursteigerung nachweisbar nicht mit irgendwelchen Vorgängen an den Genitalien und deshalb auch nicht mit der Kolpeuryse in Zusammenhang zu bringen war. Zwei Kreissende, welche im Wochenbett Temperatursteigerungen zeigten, hatten bereits beim Eintritt in die. Anstalt Fieber gehabt, also schon vor Anwendung der Braun'schen Blase. Bei 10 anderen Wöchnerinnen wurde die Störung des Wochenbetts bedingt: je zweimal durch Phthisiș pulm., Pneumonia catarrh. und Mastitis purul., und je einmal durch Influenza, Bronchitis, Cystitis und Parulis.

Bei der genauen mikroskopischen Untersuchung des Vaginalund Cervikalsecretes wurde nichts. von pathogenen Keimen gefunden, so dass eine etwa zugleich bestehende Affection der Genitalien ausgeschlossen werden konnte. Dagegen wurden bei 22 Frauen, zum Theil bereits vor der Entbindung mit Sicherbeit Gonokokken nachgewiesen, welche die Temperatursteigerungen im Wochenbett erklären, so dass dieselben auch hier nicht dem Kolpeurynter zur Last gelegt werden können.

Eihautreste waren bei 7 Wöchnerinnen die Ursache zu mässigem Fieber; nach Ausstossung derselben sank die Temperatur alsbald zur Norm zurück. Leichte Fiebersteigerungen ohne wesentliche Störung des Allgemeinbefindens, auf Staung des Lochialsecrets oder fötider. Zersetzung desselben beruhend, gab bei 31 Frauen die Veranlassung zu einer Behandlung, die in Scheiden- 
spülungen oder in Einstellung der Portio und Ablassen des gestauten Secrets bestand. Die Temperatur ging darauf hin ohne weitere Eingriffe bald zur Norm zurück.

Es bleiben nun noch 58 Frauen, die stärkere oder länger dauernde Temperatursteigerung im Wochenbett aufwiesen. In 13 Fällen fand sich eine Thrombose einer oder beider Schenkelvenen, ohne dass in den Secreten pathogene Keime nachgewiesen werden konnten; von diesen. Frauen gingen zwei an Lungenembolie zu Grunde. Auch hier kann wohl von einem Lusammenhang zwischen der Kolpeuryse und der Erkrankung oder gar dem Tode der Wöchnerinnen nicht die Rede sein. In den anderen Fällen von ernsterer fieberhafter Erkrankung im Wochenbett -.. es sind dies 45 - $\ldots$ ist wohl die Uterushöhle als Ausgangspunkt für dieselbe anzuseben, doch ist ein Todesfall unter diesen mit Kolpeuryse behandelten Fällen nicht vorgekommen.

Nehmen wir alle Fälle zusammen, bei denen das Fieber im Wochenbett durch eine Affection der Genitalien bedingt wird, so erhalten wir $16 \mathrm{pCt}$. Fieber, mit Ausschluss der nur einmalige oder geringfügige Temperatursteigerung aufweisenden Frauen sogar nur 11,5 pCt. In Anbetracht der Thatsache; dass eine grosse Zahl von Kreissenden bereits mit gesprungener Blase in die Anstalt kam und ausserdem zahlreiche Fälle von Eklampsie und Placenta praevia unter den behandelten sind, kann man wohl mit Recht sagen:

"Die vaginale Anwendung des Braun'schen Kolpeurynters beeinflusst das Wochenbett nicht ungünstig; dadurch, dass sie manche Operationen überflüssig macht, für etwa nothwendig werdende aber möglichst günstige Vorbedingungen schafft, lässt sie die Prognose für den Verlauf des Puerperiums günstiger erscheinen."

Aus eben diesem Grunde ist die Kolpeuryse von nicht zu unterschätzendem Vortheil für das Leben der Kinder. Derselbe tritt bei den Geburten bei engem Becken besonders klar zu Tage. Befinden sich doch unter den bei einer C. d. von 9,5 abwärts spontan und lebensfrisch geborenen Kindern solche von beträchtlicher Grösse: $3570 \mathrm{~g}$ und $50 \mathrm{~cm}, 3780 \mathrm{~g}$ und $52 \mathrm{~cm}$, und $4170 \mathrm{~g}$ und $56 \mathrm{~cm}$ bei Erstgebärenden und fast ebenso gut entwickelte bei Mehrgebärenden. Neun Kinder starben . unter der Geburt ab, zu einer Zeit, da die Vorbedingungen noch nicht erfüllt waren, um eine Operation zur Rettung dos Kindes zu versuchen. Nach Ausschaltung dieser Fälle bleibt noch der Tod von 8 Kindern zu verzeichnen, die asphyctisch geboren wurden und trotz sofort ange- 
stellter Wiederbelebungsversuche nicht zu regelrechtem Athmen gebracht werden konnten und bald nach der Geburt starben. Nach Ausschaltung der oben erwähnten 9 Fälle würde sich das Verhältniss der spontan bei engem Becken geborenen, lebenden Kinder zu den nicht wiederbelebten auf $222: 8$ stellen, also 3,5 pCt.

Naturgemäss stellt sich der Procentsatz an todten Kindern ganz anders bei den Fällen, die operativ beendigt. werden mussten. Bildete doch die Gefahr für die Mutter während der in Frage kommenden 6 Jahre $27 \mathrm{mal}$ die Indication dazu, das kindliche Leben durch die Perforation zu opfern. Wenn die Zahl der Perforationen des lebenden Kindes als solche gross erscheint, so muss man berücksichtigen, dass dieselbe im Verhältniss zu der Zahl der engen Becken doch recht gering ist und dass diese Thatsache doch in erster Linie durch die günstige Wirkung des Kolpeurynters zu erklären ist. Zur schonenden und schnellen Entbindung wurde ausserdem in 19 Fällen der Kopf des abgestorbenen Kindes perforirt, und zwar 9 mal bei Kopflage und $10 \mathrm{mal}$ im Anschluss an eine innere Wendung, da es nicht gelang, den nachfolgenden Kopf unzerkleinert durch das Becken zu leiten. Von den Kindern, zu deren Rettung bei engem Becken zu der inneren Wendung und Extraction geschritten wurde, kamen ausser den oben erwähnten 10 Kindern 6 tief asphyctisch zur Welt, welche trotz" sofort angestellter Wiederbelebungsversuche kurz nach der Geburt starben. Von den aus Querlage entwickelten waren 4 Kinder bereits vor der Operation abgestorben, bevor die Weichtheile für eine solche genügend vorbereitet waren. Immerhin ist auch die Zahl von 68 durch Wendung und Extraction lebend zor Welt gebrachten Kindern bei den 88 Fällen, in denen diese Operation vorgenommen wurde, als eine recht günstige zu bezeichnen. Die wesentlich schlechteren Resultate der Zangenoperationen an dem oberhalb der Beckenmitte stehenden Kopfe erklären sich wohl durch die Schwierigkeit und die lange Dauer der Operation; es konnte unter 9 Fällen nur 6 mal ein lebendes Kind entwickelt werden. Ebenso gelang es auch nicht, in jedem der - 13 - Fälle von drohender Asphyxie des Kindes, obwohl der Kopf bereits im Beckenausgang stand, das kindliche Leben zu retten, da in 4 Fällen die Kinder bereits derart gelitten hatten, dass sie trotz sofort vorgenommener Wiederbelebungsversuche kurz nach der Geburt starben. Andererseits ist aber in der geringen Zahl ron Zangenoperationen ein never Beweis dafür zu sehen, dass der Kolpeurynter mit Erfolg dazu beiträgt, 
Spontangeburten bèi engen Becken zu erzielen. Fasst man die Ergebnisse der Kolpeuryse bei engem Becken in Bezug auf die Kinder zusammen, so erhält man: bei Spontangeburten 222 lebendgeborene, 9 bereits vor der Geburt abgestorbene und 8 asphyctisch geborene und nicht wieder belebte Kinder; bei den operativen Entbindungen 151 lebend geborene, 23 vor und 46 während der Geburt abgestorbene Kinder; von den letzteren mussten 27 lebend perforirt werden.

Bei den 120 Fällen, in denen der Kolpeurynter nicht wegen engen Beckens eingelegt wurde, war acht mal das Kind bereits vor der Geburt abgestorben. An vorzeitjger Placentarlösung gingen drei Kinder unter der Geburt zu Grunde. Ein Kind, welches mit einer dreimaligen festen Nabelschnurumschlingung um den Hals tief asphyctisch geboren wurde, konnte nicht wiederbelebt werden, ebenso ein durch Zangenoperation gewonnenes, und drei durch Wendung und Extraction entwickelte Kinder. In 3 Fällen war die Perforation des todten und 7 mal die des lebenden Kindes, meist wegen ungünstiger Einstellung des Kopfes, einige Male auch wegen schwerer Eklampsie im Interesse der Mutter nothwendig. Dass trotz. der grossen Gefahr für das kindliche Leben nach einer combinirten Wendung bei Placenta praevia die Extraction zu unterlassen ist, wird fast allgemein anerkannt. Es sind deshalb bei den mit Kolpeuryse Behandelten 6 todtgeborene Kinder zu verzeichnen, welche im Interesse der Mutter geopfert werden mussten, da andere Mittel zu deren Rettung versagten.

Diese Beobachtungen bestätigen zum grossen Theil diejenigen, welche Rosenthal in der oben angeführten Arbeit niedergelegt hat; sie zeigen aber ausserdem, dass die Braun'sche Blase in noch weiterem Maasse vaginal angewendet werden kann und ein werthvolles und ungefährliches Hülfsmittel zur Leitung pathologischer Geburten ist.

Die Erfahrungen mit der vaginalen Anwendung der Braunschen Blase, welche in obiger Darstellung enthalten sind, lassen sich in folgende Sätze zusammenfassen:

Der Braun'sche Kolpeurynter, vaginal angewendet, dient

1. zur Erhaltung des Fruchtwassers, bei erhaltener, wie bei gesprungener Blase,

2. zur Vorbereitung der Weichtheile,

3. zur Verstärkung bereits vorhandener Wehen. 
Vorbedingungen für seine erfolgreiche Anwendung sind das Bestehen regelmässiger, wenn auch schwacher Wehen und die richtige Wahl in der Grösse, sowie die straffe Füllung des Ballons.

Zur Anregung von Wehen und zur Eröffnung eines narbig stenosirten Muttermundes eignet sich die vaginale Anwendung der Braun'schen Blase nicht. $\mathrm{Zu}$ einer Tamponade der Scheide bei Placenta praevia ist sie nicht so zuverlässig, wie Gazestreifen oder Wattekugeln.

Der Kolpeurynter macht eine ganze Zahl von geburtshülflichen Operationen unnöthig und schafft für die etwa nothwendig werdenden günstige Vorbedingungen; eben dieselbe Wirkung lässt seine Anwendung bei normalen Becken in geeigneten Fällen rathsam erscheinen.

Die Kolpeuryse beeinflusst den Wochenbettsverlauf nicht ungünstig, lässt im Gegentheil dadurch, dass sie geburtshülfliche Operationen unnöthig macht bezw. erleichtert, die Prognose günstiger erscheinen. Aus denselben Gründen ist sie auch für das Leben der Kinder von günstigem .Einfluss. 